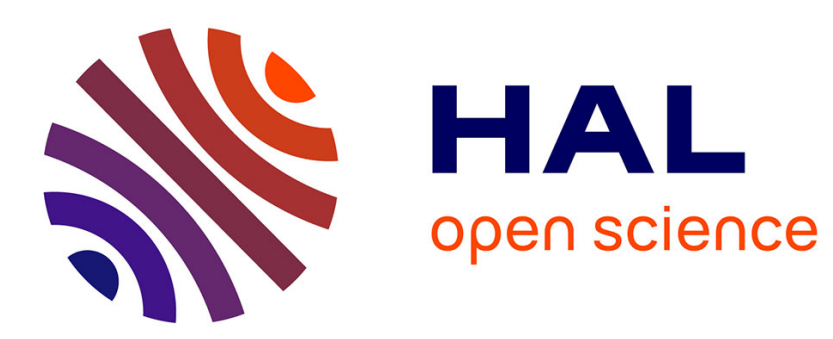

\title{
Identifying Logical Homogeneous Clusters for Efficient Wide-area Communications
}

\author{
Luiz Angelo Barchet-Estefanel, Grégory Mounié
}

\section{To cite this version:}

Luiz Angelo Barchet-Estefanel, Grégory Mounié. Identifying Logical Homogeneous Clusters for Efficient Wide-area Communications. EuroPVM/MPI 2004 11th European PVM/MPI Users' Group Meeting, 2004, France. pp.319-326. hal-00002547v3

HAL Id: hal-00002547

https://hal.science/hal-00002547v3

Submitted on 2 Dec 2004

HAL is a multi-disciplinary open access archive for the deposit and dissemination of scientific research documents, whether they are published or not. The documents may come from teaching and research institutions in France or abroad, or from public or private research centers.
L'archive ouverte pluridisciplinaire $\mathbf{H A L}$, est destinée au dépôt et à la diffusion de documents scientifiques de niveau recherche, publiés ou non, émanant des établissements d'enseignement et de recherche français ou étrangers, des laboratoires publics ou privés. 


\title{
Identifying Logical Homogeneous Clusters for Efficient Wide-area Communications
}

\author{
Luiz Angelo B. Estefanel ${ }^{\star}$ and Grégory Mounié \\ Laboratoire ID - IMAG, Project APACHE ${ }^{\star \star}$ \\ 51, Avenue Jean Kuntzmann, F-38330 Montbonnot St. Martin, France \\ \{Luiz-Angelo.Estefanel, Gregory. Mounie\}@imag.fr
}

\begin{abstract}
Recently, many works focus on the implementation of collective communication operations adapted to wide area computational systems, like computational Grids or global-computing. Due to the inherently heterogeneity of such environments, most works separate "clusters" in different hierarchy levels. to better model the communication. However, in our opinion, such works do not give enough attention to the delimitation of such clusters, as they normally use the locality or the IP subnet from the machines to delimit a cluster without verifying the "homogeneity" of such clusters. In this paper, we describe a strategy to gather network information from different local-area networks and to construct "logical homogeneous clusters", better suited to the performance modelling.
\end{abstract}

\section{Introduction}

In recent years, many works focus on the implementation of collective communications adapted to large-scale systems, like Grids. While the initial efforts to optimise such communications just simplified the models to assume equal point to point latencies between any two processes, it becomes obvious that any tentative to model practical systems should take in account the inherently heterogeneity of such systems. This heterogeneity represents a great challenge to the prediction of communication performance, as it may come from the distribution of processors (as for example, in a cluster of SMP machines), from the distance between machines and clusters (specially in the case of a computational Grid) and even from variations in the machines performance (network cards, disks, age of the material, etc.). It is also a true concern for users that run parallel applications over their LANs, where there can be combined different machines and network supports.

As the inherent heterogeneity and the growth of computational Grids make too complex the creation of full-customised collective operations, as proposed in the past by [1] 14], a solution followed by many authors is to subdivide the network in communication layers. Most systems only separate inter and intracluster communications, optimising communication across wide-area networks,

${ }^{\star}$ Supported by grant BEX 1364/00-6 from CAPES - Brazil
$\star \star$
This project is supported by CNRS, INPG, INRIA and UJF 
which are usually slower than communication inside LANs. Some examples of this "two-layered" approach include [7,9, 11, 12, where ECO [7, 11 and MagPIe [7] apply this concept for wide-area networks, and LAM-MPI 7 [9] applies it to SMP clusters. Even though, there is no real restriction on the number of layer and, indeed, the performance of collective communications can still be improved by the use of multi-level communication layers, as observed by [4. 4 .

While the separation of the network in different levels can improve the communication performance, it still needs to be well tuned to achieve optimal performance levels. To avoid too much complexity, the optimisation of two-layer communication or the composition of multiple layers relies on a good communication modelling of the network. While in this work we use pLogP [8], the main concern for the accuracy of a network model relies on the homogeneous behaviour of each cluster. If there are some nodes that behave differently from what was modelled, they will interfere with the undergoing operation. It is worth to note, however, that most of the works on network-aware collective communication seem to ignore this problem, and define clusters according to simple "locality" parameters, as for example, the IP subnet of the nodes.

While there are many network monitoring tools that could help on the identification of such heterogeneities like, for example, NWS [15], REMOS [12] or TopoMon 2, they still do not provide information about machines that hold multiple application processes, like SMP machines. Further, these tools are unable to identify heterogeneities due to the application environment, as for example, the use of an IMPI [3] server to interconnect different MPI distributions, or an SSH tunnel among different clusters protected by a firewall.

In this paper, we describe a framework to allow the gathering of independent network information from different clusters and the identification of "logical clusters". Our proposal combines the detection of "homogeneity islands" inside each cluster with the detection of SMP processors, allowing the stratification of the network view, from the node layer (specially in the case of SMP machines) to the wide-area network.

Section 2 presents our proposal for automatic topology discovery. The framework is divided in two phases. The first one, presented on Section [3, explains how connectivity data collected by different clusters can be put together. Section 19 presents the second phase, which explains how "logical clusters" are defined from the collected data, and how SMP nodes can be identified. Section 0 the results from a practical experiment, and some considerations on the benefits from the use of our framework. Finally, Section 6 presents our conclusions and perspective for future works.

\section{What we propose}

We propose a method to automatically discover network topology in order to allow the construction of optimised multilevel collective operations. We prefer automatic topology discovery instead of a predefined topology because if there are hidden heterogeneities inside a cluster, they may interfere with the communication and induce a non negligible imprecision in the communication models. 
The automatic discovery we propose should be done in two phases: the first phase collects reachability data from different networks. The second phase, executed at the application startup, identifies SMP nodes (or processes in the same machine), subdivides the networks in homogeneous clusters and acquires pLogP parameters to model collective communications.

As the first step is independent from the application, it can use information from different monitoring services, which are used to construct a distance matrix. This distance matrix does not need to be complete, in the sense that a cluster does not need to monitor its interconnection with other clusters, and several connectivity parameters can be used to classify the links and the nodes as, for example, latency and throughput.

When the network is subdivided in homogeneous subnets, we can acquire pLogP parameters, necessary to model the collective communications and to determine the best communication schedule or hierarchy. Due to the homogeneity inside each subnet, pLogP parameters can be obtained in an efficient way, which reflects in a small impact on the application initialisation time.

At the end of this process we have logical clusters of homogeneous machines and accurate interconnection parameters, that can be used to construct an interconnection tree (communicators and sub-communicators) that optimises both inter and intra-cluster communication.

\section{First Phase: Gathering Network Information}

While there are many works that focus on the optimisation of collective communications in Grid environments, they consider for simplicity that a cluster is defined by its locality or IP subnet, and that all machines inside a cluster behave similarly. Unfortunately, this "locality" assumption is not adequate to real systems, which may contain machines that behave differently both in performance and in communication. In fact, even in clusters with similar material, machines can behave differently (we believe that it is nearly impossible to have homogeneity in a cluster with hundreds of machines). Hence, to better optimise collective communications in a Grid environment, the choice of the topologies must be based on operational aspects that reflect the real performance level of each machine or network.

\subsection{Obtaining Network Metrics}

There are many tools specialised on network monitoring. These tools can obtain interconnectivity data from direct probing, like for example NWS [15], from SNMP queries to network equipments, like REMOS 12], or even combine both approaches, like TopoMon [2. For simplicity, this work obtains data at the application level, with operations built according to NWS definition. We chose NWS as it is a de facto standard in the Grid community, and can be configured to provide information like communication latency, throughput, CPU load and available memory. To our interest, we can use communication latency and 
throughput, obtained from NWS, to identify sets of machines with similar communication parameters.

However, contrarily to some tools like TopoMon, our method does not require total interconnection among all nodes in all clusters. Indeed, the objective of the first step of our topology discovery is to identify heterogeneity inside each cluster, and by this reason, each cluster may use its own monitoring tool, without being aware of other clusters. This strategy allows the use of regular monitoring data from each cluster, while does not create useless traffic between different clusters. Hence, the data obtained from different clusters is collected and used to construct a distance matrix, which will guide the elaboration of the cluster hierarchy for our collective operations.

As clusters are not aware of each other, the "missing interconnections" clearly delimit their boundaries, which reduces the cost of the clustering process. Moreover, we are not strongly concerned with the problem of shared links, like [2] or [10, because the reduction of the number of messages exchanged among different clusters is part of the collective communication optimisation.

\section{Second Phase: Application-level Clustering}

One reason for our emphasis on the construction of logical clusters is that machines may behave differently, and the easiest way to optimise collective communications is to group machines with similar performances. In the following section we describe how to separate machines in different logical clusters according to the interconnection data we obtained in the First Phase, how to identify processes that are in the same machine (SMP or not), and how this topology knowledge may be used to obtain pLogP parameters in an efficient way.

\subsection{Clustering}

From the interconnection data from each cluster acquired on the previous phase, we can separate the nodes in different "logical cluster". To execute this classification, we can use an algorithm similar to the Algorithm 1, presented by ECO 12.

This algorithm analyses each interconnection on the distance matrix, grouping nodes for wich their incident edges respect a latency bound ( $20 \%$, by default) inside that subnet. As this algorithm does not generate a complete hierarchy, just a list of subnets, it does not impose any hierarchical structure that would "freeze" the topology, forbidding the construction of dynamic inter-cluster trees adapted to each collective communication operation and its parameters (message size, segments size, etc.).

\subsection{SMP Nodes and Group Communicators}

While NWS-like tools provide enough information to identify logical clusters, they cannot provide information about processes in SMP nodes, as they are created by the application. Actually, as the processes distribution depends on 


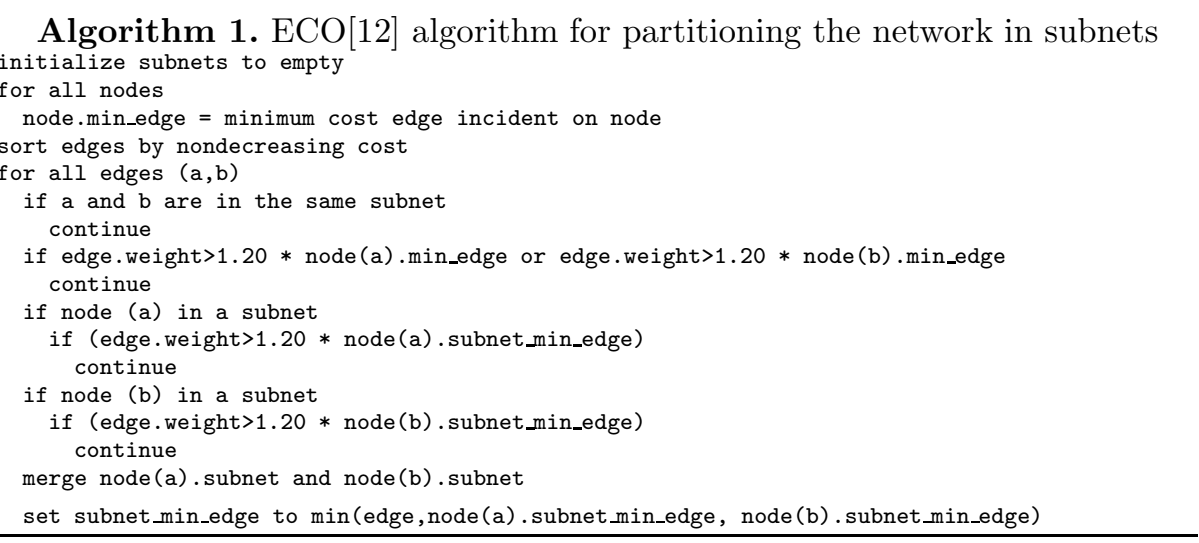

the application parameters (and environment initialisation), the identification of processes in SMP nodes shall be done during the application startup.

However, the implementation of an SMP-aware MPI is not easy, because the definition of MPI does not provides any procedure to map process ranks into real machine names. To exemplify this difficulty, we take as example the recent version 7 from LAM/MPI 9]. Their SMP aware collective communications, based on MagPIe [7], rely on the identification of processes that are started in the same machine, but they use proprietary structures to identify the location of each process. To avoid be dependent on a single MPI distribution, we adopted a more general solution, where each process, during its initialisation, call gethostname(), and sends this data to a "root" process that will centralise the analysis. If perhaps this approach is not as efficient as the one used by LAM, it still allows the identification of processes in the same machine (what can be assumed as an SMP machine).

As the data received by the root contains both the machine name and the process rank, it can translate the logical clusters into communicators and subcommunicators, adapted to the MPI environment.

\section{Practical Results}

\subsection{Clustering}

To validate our proposal, we looked for a network environment that could raise some interesting scenarios to our work. Hence, we decided to run our tests on our experimental cluster, IDPOT" ${ }^{1}$. IDPOT can be considered as a "distributed cluster", as its nodes are all spread through our laboratory, while connected with a dedicated Gigabit Ethernet (two switches). All machines are Bi-Xeon 2.5 $\mathrm{GHz}$, with Debian Linux 2.4.26, but they have network card from two different manufacturers, and the distribution of the machines in the building may play

\footnotetext{
${ }^{1}$ http://idpot.imag.fr
} 
an important role in the interconnection distance between them and the Gigabit switches.

Applying the methods described in this paper over a group of 20 machines from IDPOT gives the following result, depicted on Fig. 1. This figure presents the resulting subnets, as well as the interconnection times between each subnet and among nodes in the same subnet. It is interesting to note how the relative latency among each cluster would affect a collective communication that was not aware of such information. For example, in the case of a two-level model, subnets $\mathrm{C}$ and $\mathrm{E}$ would affect the expected performance, as their interconnections are twice or even three times slower than others. This would also reflect in the case of a multi-layer model, where an unaware algorithm could prefer to connect directly subnets $\mathrm{C}$ and $\mathrm{E}$, while it is more interesting to forward communications through subnet D.

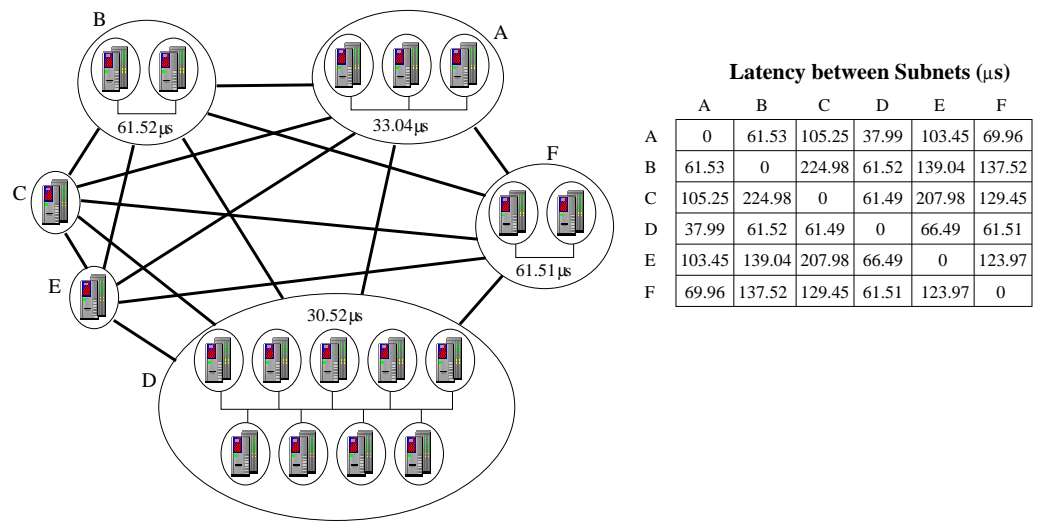

Figure1. IDPOT network partition, with latency among nodes and subnets

We identified as the main factor for such differences the presence of network cards from one manufacturer on subnets A and D, while subnets B, C, E and F have onboard cards from other manufacturer. As second factor, we can list the location of the nodes. While it played a less important role, the location was the main cause for separation between subnet A and subnet D. Actually, the distance between those machines, which are under different switches, affected the latency just enough to force ECO's algorithm to separate them in two different subnets. A correct tuning on the parameters from ECO's algorithm may allow subnets A and $\mathrm{D}$ to be merged in a single one, a more interesting configuration for collective communications.

\subsection{Efficient Acquisition of pLogP Parameters}

While the logical clusters generated by our framework allow a better understanding of the network effective structure, we are still unable to model communications with precision. This first reason is that interconnection data may be incomplete. As said in Section 3.1, the monitoring tools act locally to each LAN, and by this reason, they do not provide data from the inter-cluster connections. 
Besides this, the data acquired by the monitoring tools is not the same as the data used in our models. For example, the latency, which originally should have the same meaning to the monitoring tool and the application, is obtained differently by NWS and pLogP. In NWS, the latency is obtained directly from the round-trip time, while pLogP separates the round-trip time in latency and gap, as depicted by Figure 2, with differences that may interfere on the communication model. In addition, the information collected by the monitoring tools is not obtained by the application itself, and thus, is not submitted to the same constraints that the application will find at runtime, as for example, the use of an Interoperable MPI (IMPI) server to interconnect the clusters.
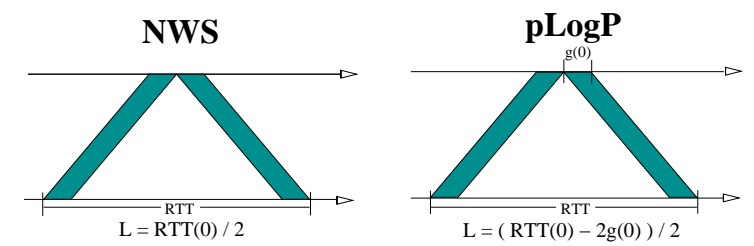

Figure2. Differences between NWS and pLogP "latency"

Hence, to model the communication in our network, we need to obtain parameters specifically for pLogP. Hopefully, there is no need to execute $n(n-1)$ pLogP measures, one for each possible interconnection. The first reason is that processes belonging to the same machine were already identified as SMP processes and grouped in specific sub-communicators. And second, the subnets are relatively homogeneous, and thus, we can get $\mathrm{pLogP}$ parameters in an efficient way by considering a single measure inside each subnet as a sample from the pLogP parameters common to the entire cluster. As one single measure may represents the entire subnet, the total number of pLogP measures is fairly reduced. If we sum up the measures to obtain the parameters for the inter-clusters connections, we shall execute at most $\mathrm{C}(\mathrm{C}+1)$ experiments, where $\mathrm{C}$ means the number of subnets. Further, if we suppose symmetrical links, we can reduce this number of measures by half, as $a \rightarrow b=b \rightarrow a$. By consequence, the acquisition of pLogP parameters for our experimental 20-machines cluster would need at most $(6 *(6+1)) / 2=21$ measures.

\section{Conclusions}

This paper proposes a simple and efficient strategy to identify communication homogeneities inside computational clusters. The presence of communication heterogeneities reduces the accuracy from the communication models used to optimise collective communications in wide-area networks. We propose a low cost method that gathers connectivity information from independent clusters and groups nodes with similar characteristics. Using real experiments on one of our clusters, we show that even minor differences may have a direct impact on the communication performance. Our framework allowed us to identify such differences, classifying nodes accordingly to their effective performance. Using such 
classification, we can ensure a better accuracy for the communication models, allowing the improvement of collective communication performances, specially those structured on multiple layers.

\section{References}

1. Bhat, P., Raharendra, C., Prasanna, V.: Efficient Collective Communication in Distributed Heterogeneous Systems. Journal of Parallel and Distributed Computing, No. 63, Elsevier Science. (2003) 251-263

2. Burger, M., Kielmann, T., Bal, H.: TopoMon: a monitoring tool for Grid network topology. Intl. Conference on Computational Science'02. Springer-Verlag, LNCS Vol. 2330 (2002) pp. 558-567.

3. Interoperable MPI Web page. http://impi.nist.gov

4. Karonis, N. T., Supinski, B., Foster, I., Gropp, W., Lusk, E., Bresnahan, J.: Exploiting Hierarchy in Parallel Computer Networks to Optimize Collective Operation Performance. In: 14th International Conference on Parallel and Distributed Processing Symposium. IEEE Computer Society (2000) 377-384.

5. Karonis, N. T., Foster, I., Supinski, B., Gropp, W., Lusk, E., Lacour, S.: A Multilevel Approach to Topology-Aware Collective Operations in Computational Grids. Technical report ANL/MCS-P948-0402, Mathematics and Computer Science Division, Argonne National Laboratory (2002).

6. Karonis, N. T., Toonen, B., Foster, I.: MPICH-G2: A Grid-enabled implementation of the Message Passing Interface. Journal of Parallel and Distributed Computing, No. 63, Elsevier Science. (2003) 551-563

7. Kielmann, T., Hofman, R., Bal, H., Plaat, A., Bhoedjang, R.: MagPIe: MPI's Collective Communication Operations for Clustered Wide Area Systems. In: 7th ACM SIGPLAN Symposium on Principles and Practice of Parallel Programming, ACM Press. (1999) 131-140

8. Kielman, T., Bal, E., Gorlatch, S., Verstoep, K, Hofman, R.: Network Performanceaware Collective Communication for Clustered Wide Area Systems. Parallel Computing, Vol. 27, No. 11, Elsevier Science. (2001) 1431-1456

9. LAM-MPI Team, LAM/MPI Version 7, http://www.lam-mpi.org/ (2004)

10. Legrand, A., Quinson, M.: Automatic deployment of the Network Weather Service using the Effective Network View. In: High-Performance Grid Computing Workshop (associated to IPDPS'04), IEEE Computer Society (2004)

11. Lowekamp, B., Beguelin, A.: ECO: Efficient Collective Operations for communication on heterogeneous networks. In: 10th International Parallel Processing Symposium. (1996) 399-405

12. Lowekamp, B.: Discovery and Application of Network Information. PhD Thesis, Carnegie Mellon University. (2000)

13. Thakur, R., Gropp, W.: Improving the Performance of Collective Operations in MPICH. In: Euro PVM/MPI 2003, Springer-Verlag, LNCS Vol. 2840 (2003) 257267.

14. Vadhiyar, S., Fagg, G., Dongarra, J.: Automatically Tuned Collective Communications. In: Supercomputing 2000, Dallas TX. IEEE Computer Society (2000)

15. Wolski, R., Spring, N., Peterson, C.: Implementing a Performance Forecasting System for Metacomputing: The Network Weather Service. In: Supercomputing 1997 (1997) 